\title{
Radio Physics and Radio Astronomy
}




\section{Radio Physics and Radio Astronomy}

Volume 3/Issue 1

2012

RADIO ASTRONOMY AND ASTROPHYSICS

Heliograph of the UTR-2 Radio Telescope. III. Observations

1

A. A. Konovalenko, A. A. Stanislavsky, A. A. Koval, \& E. P. Abranin

Focusing of the Quasar Radiation in the Gravitational Field of a Microlens-Star Located in a Macrolens-Galaxy: 2. Isophot Structure and Amplification of Brightness of the Radiation Source Visible Image

L. A. Berdina, A. A. Minakov, \& V. G. Vakulik

Observation of ${ }^{13} \mathrm{CO}$ and Methanol Line Emission

from Supernova Remnant Kes 79

V. M. Shulga, S. Y. Zubrin, \& V. V. Myshenko

Bipolar Molecular Outflows in the Star Forming Region IRAS 22267+6244

A. V. Antyufeyev \& V. M. Shulga

PROPAGATION, DIFFRACTION AND SCATTERING OF ELECTROMAGNETIC WAVES

Inverse Transmission Problem for Plane Electromagnetic Wave Normally

Incident through a Layered Dielectric Structure

Z. T. Nazarchuk, R. O. Hryniv, \& A. T. Synyavskyy

Evaluation of Gaussian beam Scattering Characteristics for Double-Periodic Structures

A. V. Gribovsky \& O. A. Yeliseyev

Operator Method in Problem of Radiation through a System of Slots in a Planar Waveguide

M. E. Kaliberda \& S. A. Pogarsky

Plane $\boldsymbol{H}$-Polarized Electromagnetic Wave Scattering

by Infinite Dihedral Wedge Co-Axially Coupled with Slotted Cylinder

Y. V. Shepilko

\section{RADIO SPECTROSCOPY}

Excitation of Lead Atoms to Rydberg States

S. F. Dyubko, N. L. Pogrebnyak, A. S. Kutsenko, \& M. P. Perepechay

Millimeter- and Submillimeter-Wave Spectrometers on the Basis of Direct Digital Frequency Synthesizers

E. A. Alekseev, R. A. Motiyenko, \& L. Margulès 


\section{Radio Physics and Radio Astronomy}

Volume 3/Issue 2

2012

RADIO ASTRONOMY AND ASTROPHYSICS

Using Phase Dynamic Cross-Spectra for Wideband

Radio Astronomy Observations: Experience

from the UTR-2 Radio Telescope

89

V. L. Koliadin

A Theory of Radiation by a Relativistic Charged Particle

Moving through a Curved Magnetic Field:

The Undulator-Curvature Mechanism

103

Ya. M. Sobolev

Investigation of Large-Scale Structure of Solar Wind at Spatially Separated Radiotelescopes

113

M. R. Olyak

Parameter Determination of Kiloparsec Jets at Large Redshifts

by Their Radio and X-Ray Emission

M. S. Butuzova

Scintillations of Radio Source 4C21.53 at Decameter Wavelengths

and Elongations $\mathbf{4 3}^{\circ}-\mathbf{1 3 8}^{\circ}$

131

N. N. Kalinichenko

\section{RADIO PHYSICS OF GEOSPACE}

Comparative Analysis of Ionospheric Effects as Observed during "Proton" Rocket Flights under Different Space Weather Conditions

L. F. Chernogor \& T. G. Zhivolup

PROPAGATION, DIFFRACTION AND SCATTERING OF ELECTROMAGNETIC WAVES

Dispersion Properties of Reflecting Grating Filled with Metamaterial

O. P. Kusaykin, P. N. Melezhik, S. B. Panin, \& A. Y. Poyedynchuk

\section{RADIO SPECTROSCOPY}

Microwave Spectroscopy of Torsion Vibrations in Molecules:

Approximation of Strict Coupling between the Structural Torsional Parameters $\rho$ and $F$ in the Rho Axis Method

V. V. Ilyushin 
RADIO PHYSICAL PROBLEMS OF RADAR, RADIO NAVIGATION, COMMUNICATIONS AND REMOTE SENSING

Multi-Look Radiometric Correction of SAR Images

169

O. O. Bezvesilniy, I. M. Gorovyi, V. V. Vynogradov, \& D. M. Vavriv

MICROWAVE PHENOMENA IN DEVICES, COMPONENTS

AND SYSTEMS OF SCIENTIFIC INSTRUMENTS

Unsaturated Mode as Alternative Method to Provide Stability

of Low-Noise Amplifiers on High-Electron-Mobility Transistors

179

A. M. Korolev \& V. M. Shulga 


\section{Radio Physics and Radio Astronomy}

Volume 3/Issue 3

2012

RADIO ASTRONOMY AND ASTROPHYSICS

Peculiarity of Continuum Emission from the Upper Solar Corona at Decameter Wavelengths

A. I. Brazhenko, A. A. Koval, A. A. Konovalenko, A. A. Stanislavsky,

E. P. Abranin, V. V. Dorovskyy, V. N. Melnik, R. V. Vashchishin,

A. V. Frantzusenko, \& O. V. Borysyuk

Inverse Compton and Thomson Scattering of the Quasar 3C 273 Emission by Electrons of the Nearest Knot of Its Kiloparsec Jet

M. S. Butuzova

Propagation of Type III Solar Bursts Radio Emission through the Corona.

1. Time Profile

B. P. Rutkevych \& V. N. Melnik

Physical Parameters of Radio Sources with Steeply Rising Decameter Wavelength Spectra

A. P. Miroshnichenko

Mechanism of Degradation of Images Observed through a Medium with Random Inhomogeneities

Y. V. Kornienko \& S. I. Skuratovskiy

\section{RADIO PHYSICS OF GEOSPACE}

Background Variations in the Total Electron Content of the Ionosphere over the Antarctic Peninsula

A. A. Sopin, Y. M. Zanimonskiy, V. N. Lisachenko, \& Y. M. Yampolski

Geomagnetic Pulsations Associated with the Solar Terminator Motion through the Magnetoconjugate Region

L. F. Chernogor

Searching the "Weekend Effect" in Intensity Variations of the Natural ELF Noise

A. V. Paznukhov, Y. M. Yampolski, Y. M. Zanimonskiy, \& A. V. Soina

PROPAGATION, DIFFRACTION AND SCATTERING OF ELECTROMAGNETIC WAVES

Operator Method in the Problem of Radiation from an Open End

of a Circular Corrugated Waveguide

M. E. Kaliberda, S. A. Pogarsky, \& V. A. Belousov 
Diffraction Effects in Cylindrical Monopole and Dielectric Disk Antennas

I. V. Ivanchenko, N. A. Popenko, \& M. M. Khruslov

RADIO PHYSICAL PROBLEMS OF RADAR, RADIO NAVIGATION, COMMUNICATIONS AND REMOTE SENSING

Dealiasing Doppler Spectra in Meteorological Radars

273

S. V. Sosnytskiy 


\section{Radio Physics and Radio Astronomy}

Volume 3/Issue 4

RADIO ASTRONOMY AND ASTROPHYSICS

An Unusual Solar Burst at Decameter Wavelengths. 1. Observations

A. I. Brazhenko, V. N. Melnik, A. A. Konovalenko, V. V. Dorovskyy,

A. V. Frantsuzenko, H. O. Rucker, M. Panchenko, \& A. A. Stanislavsky

Propagation of Type III Solar Bursts Radio Emission through the Corona. 2. Frequency Drift Rate

B. P. Rutkevych \& V. N. Melnik

\section{RADIO PHYSICS OF GEOSPACE}

A Possible Mechanism of the "Self-Scattering Effect" of Powerful HF Signals in the Ionosphere

V. G. Galushko, V. G. Bezrodny, A. V. Koloskov, \& A. V. Zalizovski

On the Possibility of Recovering Spatial Distribution of Ionospheric Inhomogeneities Using Data from Imaging Riometers

O. V. Charkina

Dynamics of the Ionospheric Plasma above Kharkiv during the January 4, 2011 Solar Eclipse

I. F. Domnin, L. Ya. Emelyanov, \& L. F. Chernogor

\section{PROPAGATION, DIFFRACTION AND SCATTERING OF ELECTROMAGNETIC WAVES}

Radiation from a Slot Array in a Coaxial Line Screen

V. A. Katrich, V. A. Lyashchenko, \& N. V. Medvedev

\section{STATISTICAL RADIO PHYSICS}

Near Field-to-Far Field Relation in the Problem of Plane Wave Scattering by a Statistically Rough Surface

A. S. Bryukhovetski

\section{RADIO SPECTROSCOPY}

Laser-Microwave Spectroscopy of Singlet F-Terms of the Mg I Atom 
ANTENNAS, WAVEGUIDES AND QUASIOPTICS

Non-Redundant Antenna Configurations on a 2-D Interferometer Aperture Completely Covering Central Domains in the Frequency Space

L. E. Kopilovich

RADIO PHYSICAL PROBLEMS OF RADAR, RADIO NAVIGATION, COMMUNICATIONS AND REMOTE SENSING

Local-Quadratic Map-Drift Autofocus for Synthetic Aperture Radars

O. O. Bezvesilniy, I. M. Gorovyi, \& D. M. Vavriv 


\section{AUTHOR INDEX TO VOLUME 3}

\section{Radio Physics and Radio Astronomy}

Page Ranges of Issues

Issue 1: 1-88; Issue 2: 89-185;

Issue 3: 187-278; Issue 4: 279-375

Abranin, E.P., 1, 187

Alekseev, E.A., 75

Antyufeyev, A.V., 27

Belousov, V.A., 259

Berdina, L.A., 7

Bezrodny, V.G., 291

Bezvesilniy, O.O., 169,365

Borysyuk O.V., 187

Brazhenko, A I., 187,279

Bryukhovetski, A.S., 337

Butuzova, M.S., 123,197

Charkina, O.V., 303

Chernogor L.F., 139,241,311

Domnin, I.F., 311

Dorovskyy, V.V., 187,279

Dyubko, S.F., 67

Emelyanov, L.Ya., 311

Frantsuzenko, A.V., 187,279

Galushko, V.G., 291

Gorovyi, I.M., 169,365

Gribovsky, A.V., 45

Hryniv, R.O., 33

Ilyushin, V.V., 159

Ivanchenko, I.V., 265

Kaliberda, M.E., 55,259

Kalinichenko, N.N., 131
Katrich, V.A., 325

Khruslov, M.M., 265

Koliadin, V.L., 89

Koloskov, A.V., 291

Konovalenko, A.A., 1,187,279

Kopilovich, L.E., 359

Kornienko, Y.V., 223

Korolev, A.M., 179

Koval , A.A., 1,187

Kusaykin, O.P., 149

Kutsenko, A.S., 67,353

Lisachenko, V.N., 233

Lyashchenko, V.A., 325

Margulès, L., 75

Medvedev, N.V., 325

Melezhik, P.N., 149

Melnik, V N., 187,205,279,285

Minakov, A.A., 7

Miroshnichenko, A.P., 215

Motiyenko, R.A., 75

Myshenko, V.V., 19

Nazarchuk, Z.T., 33

Olyak, M.R., 113

Panchenko, M., 279

Panin, S.B., 149

Paznukhov, A.V., 251
Perepechay, M.P., 67

Pogarsky, S.A., 55,259

Pogrebnyak, N.L., 67

Popenko, N.A., 265

Poyedynchuk, A.Y., 149

Rucker, H.O., 279

Rutkevych, B.P., 205,285

Shepilko, Y.V., 61

Shulga, V.M., 19,27,179

Skuratovskiy, S.I., 223

Sobolev, Y.M., 103

Soina, A.V., 251

Sopin, A.A., 233

Sosnytskiy, S.V., 273

Stanislavsky, A.A., 1,187,279

Synyavskyy, A.T., 33

Vakulik, V.G., 7

Vashchishin, R.V., 187

Vavriv, D.M., 169,365

Vynogradov, V.V., 169

Yampolski, Y.M., 233,251

Yeliseyev, O.A., 45

Zalizovski, A.V., 291

Zanimonskiy, Y.M., 233,251

Zhivolup, T.G., 139

Zubrin, S.Y., 19 


\title{
SUBJECT INDEX TO VOLUME 3
}

\author{
Radio Physics and Radio Astronomy
}

Page Ranges of Issues

Issue 1: 1-88; Issue 2: 89-185;

Issue 3: 187-278; Issue 4: 279-375

(u, v)-frequency space, 359

active regions, 279

airborne SAR, 169

aliasing, 273

annular slot,325

anthropogenic activity, 251

arc slot, 325

aspect-sensitive scattering, 291

auroral oval, 303

autofocus, 365

bilinear interpolation, 303

bipolar outflow, 27

Bragg reflection, 149

coaxial line, 325

correlation function, 311

corrugated waveguides, 259

cross-polarization function, 1

curvature radiation mechanism, 103

cylinder, 61

cylindrical monopole antenna, 265

decameter wavelengths, 131,279

Delaunay triangulation, 303

dielectric disk antenna, 265

diffraction, 265

digital spectrum analyzer, 131

dihedral wedge, 61

direct digital frequency synthesizer, 75

discrete cosmic sources, 303

Doppler spectra, 273

double-periodic structures, 45

duration, 279

dynamic processes, 311

dynamic spectrum, 89

electrodynamic characteristics, 45

electromagnetic wave, 61

energy coefficients, 325

excited states, 67

field-aligned irregularities, 291

Fraunhofer diffraction, 337

frequency dispersion, 149

frequency drift rate, 285

frequency drift, 279

Fresnel zone, 337

Fresnel-size inhomogeneities, 303 galaxies, 215

geomagnetic pulsations, 241

geospace plasma, 311

GNSS, 233

gravitational microlensing,7

group delay, 205

group velocity, 205,285

heliograph, 1

HEMT, 179

hindered internal rotation, 159

horn antennas, 259

image isophots, 7

inhomogeneous medium, 223

integral of a rapidly oscillating function, 337

interference, 265

interferometer, 359

internal gravity waves, 139

interplanetary medium, 113

interplanetary scintillations, 131

inverse Compton scattering, 123,197

inverse problem, 33

ionization potential, 67

ionosphere, 291

ionospheric disturbances, 139

ionospheric inhomogeneities, 233

ionospheric interlayer duct channel, 291

ionospheric refraction, 291

ionsopheric scintillations,303

jet to line of sight angle, 123

Kes 79, 19

kiloparsec jet, 123,197

laser excitation, 67

layered dielectric, 33

lead, 67

longitudinal slot, 61

low-noise, 179

luminosity, 215

macrolens-galaxy, 7

magnesium, 353

magnetic storm, 139

magnetoacoustic waves, 241

magnetoconjugate region, 241

magnetohydrodynamic waves,139

magnetomotive force method, 325 
magnification factor, 7

maser radiation, 19

metamaterial, 149

meteorological radar, 273

methanol, 19

microlens-star, 7

microwave spectroscopy, 159

microwave spectrum, 353

millimeter wavelength ranges, 75

molecular cloud, 19,27

motion error compensation, 365

motion errors, 365

motion velocity, 311

multi-look processing, 169

non-redundant configuration, 359

one-dimensional heliograph, 187

operator equations, 55,259

patterns, 45

periodic waveguide, 149

phase accumulation, 223

phase problem, 223

phase recovery, 33

phase spectrum, 89

planar difference set, 359

planar waveguide, 55

plasma generation mechanism, 279

pulsar, 103

quasar $1745+624,123$

quasar 3C 273, 197

quasar PKS 1127-14, 123

quasar, 7,215

quiet Sun, 1,187

radio and $\mathrm{X}$-ray emission, 123

radio astronomy, 89

radio emission flux density, 187

radio emission, 279

radio frequency emission, 205,285

radio frequency spectrum, 215

radio interferometry, 89

radio telescopes, 89

radio wave incoherent scattering technique, 311

radiometric correction, 169

radiometric errors, 169

refraction index inhomogeneities, 223

Rho axis method, 159

riometer, 303 rocket launches, 139

rotational spectrum, 75

Rydberg atom, 67

Rydberg atoms laser excitation, 353

scattering, 61

Schumann resonator, 251

scintillation cross-spectrum, 113

scintillation index, 303

scintillation spectrum, 113

self-scattering effect, 291

semiinfinite structure, 55

signal processing, 89

Sobolev method, 7

solar eclipse, 311

solar terminator, 241

spectral index, 187,215

spectrometer, 75

spectrum analyzer, 1

spectrum of interferometer response power fluctuations, 113

stability, 179

stationary phase, 337

statistical analysis, 241

submillimeter wavelength ranges, 75

Sun, 279

supernova remnants, 19

surface and leaking waves, 149

synchro-curvature radiation, 103

synthetic aperture radar (SAR), 169,365

three-dimensional Gaussian beam, 45

thunderstorm activity, 251

time profile, 205,285

torsion-rotation spectrum, 159

Total Electron Content, 233

transition frequency, 67

transmission coefficient, 33

transverse slot array, 325

type III bursts, 205,285

undulator radiation, 103

unusual burst, 279

upper corona, 187

UTR-2 radio telescope, 1

wave scattering, 337

weekend effect, 251

weekly variations, 251

$\mathrm{X}$-ray emission, 197 